\title{
Cation Association with Sodium Dodecyl Sulfate Micelles As Seen by Lanthanide Luminescence
}

\author{
María José Tapia, ${ }^{\dagger}$ Hugh D. Burrows, ${ }^{*}$, M. Emília D. G. Azenha, ${ }^{\ddagger}$ M. da Graça Miguel,, \\ A. A. C. C. Pais, ${ }^{\ddagger}$ and J. M. G. Sarraguça \\ Departamento de Química, Universidad de Burgos, Plaza Misael Bañuelos s/n, Burgos 09001, Spain, and \\ Departamento de Química, Universidade de Coimbra, 3004-535 Coimbra, Portugal
}

Received: November 8, 2001

\begin{abstract}
The interaction of trivalent lanthanides with sodium dodecyl sulfate micelles (SDS) in aqueous solution has been studied by a variety of experimental techniques. Potentiometric measurements with a sodium selective electrode, steady-state fluorescence spectra of $\mathrm{Ce}(\mathrm{III})$, emission lifetime measurements of $\mathrm{Ce}$ (III), $\mathrm{Tb}$ (III), and $\mathrm{Eu}(\mathrm{III})$, and electronic paramagnetic resonance spectra (EPR) of Gd(III) all show that the lanthanide ions bind to the micellar surface. From analysis of the $\mathrm{Tb}(\mathrm{III})$ lifetime measurements in water and $\mathrm{D}_{2} \mathrm{O}$ solutions, it is found that the lanthanide ions lose one hydration water on binding to SDS. However, the EPR measurements suggest that the lanthanide ions still have considerable freedom of movement. Energy transfer between $\mathrm{Ce}(\mathrm{III})$ and $\mathrm{Tb}(\mathrm{III})$ has been used to obtain further information on multiple lanthanide ion binding. From steady-state fluorescence measurements in aqueous solution in the absence of SDS no energy transfer is observed, although there is quenching of $\mathrm{Tb}(\mathrm{III})$ emission by $\mathrm{Ce}(\mathrm{III})$, which is found to follow good SternVolmer kinetics. In the presence of SDS micelles, very different behavior is observed and energy transfer occurs from excited $\mathrm{Ce}(\mathrm{III})$ to $\mathrm{Tb}$ (III). This is only possible when the two ions are on the same micelle. The energy transfer phenomena is highly dependent on micelle concentration and has been analyzed theoretically via a Monte Carlo simulation. This shows that the lanthanide ions bind close to the micelle surface, and are consistent with the loss of a water molecule. Also, assuming a Dexter-type model in which the energy transfer intensity is proportional to the inverse of the square root of the average distance between cerium and the closest terbium it is possible to reproduce qualitatively the experimental cerium(III)-sensitized $\mathrm{Tb}$ (III) luminescence intensity data.
\end{abstract}

\section{Introduction}

The interaction of cations with anionic surfactants in aqueous solutions is of both considerable theoretical ${ }^{1-7}$ and practical ${ }^{8,9}$ importance, and can have dramatic effects on the phase behavior. Of particular importance is the interaction of divalent and trivalent metal ions, such as calcium(II). Addition of these ions to common anionic amphiphiles, like sodium dodecyl sulfate (SDS), leads to reduction in solubility ${ }^{10,11}$ and an increase in Krafft temperature. ${ }^{12}$

Various methods have been used to study the interaction of cations with anionic surfactants, including surface tension, ${ }^{11,13}$ electrical conductivity, ${ }^{14}$ potentiometry, ${ }^{15,16}$ nuclear magnetic resonance ${ }^{17,18}$ and electron spin resonance ${ }^{19}$ spectroscopy, selfdiffusion measurements, ${ }^{20}$ ultrafiltration, ${ }^{21}$ ellipsometry and second-harmonic generation, ${ }^{13}$ time-resolved fluorescence quenching (TRFQ), ${ }^{22}$ and micellar catalysis. ${ }^{9}$

Trivalent lanthanide ions have attractive spectroscopic and magnetic properties. ${ }^{23}$ They possess many similarities in their properties to calcium(II) and have been used as models for its behavior. ${ }^{24}$ Of particular relevance is their luminescence, which normally arises from $f \rightarrow f$ electron transitions, and which can give information on both coordination environment ${ }^{25-27}$ and the

* Author to whom correspondence should be addressed. Fax: 351+239+827703. E-mail: burrows@ci.uc.pt

$\dagger$ Universidad de Burgos.

$\doteqdot$ Universidade de Coimbra. degree of hydration ${ }^{28,29}$ of these ions. Luminescence, because of its high sensitivity, has become one of the most popular techniques of studying surfactant association in solution. ${ }^{30-34}$ There is increasing interest in long-lived luminescent probes, ${ }^{31,34,35}$ and lanthanide ions would appear to be good candidates for this, particularly as their emission is not quenched by oxygen. However, the lanthanide $f \rightarrow f$ transitions are generally forbidden by both spin and Laporte selection rules, and hence have low molar absorption coefficients. ${ }^{36,37}$ In contrast, with cerium(III) the lowest energy electronic band in absorption corresponds to the allowed $4 f \rightarrow 5 d$ transition. Although this results in a much broader band than with the other trivalent lanthanides, it does mean that the transition has a reasonable molar absorption coefficient. ${ }^{38,39}$ In addition, energy transfer is possible to other lanthanides, such as Eu(III) and Tb(III). ${ }^{40}$ Both dipole-dipole

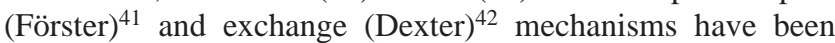
suggested for energy transfer between lanthanide ions. ${ }^{40,43}$ However, in both cases, this requires relatively close contact, and is favored by complexation ${ }^{44}$ and binding of complexes of the ions to charged species, such as micelles, ${ }^{43,45}$ phospholipid vesicles, ${ }^{46}$ or various other biologically relevant systems. ${ }^{47}$

The association of lanthanide ions with surfactant aggregates also has other applications and implications. For example, systems of lanthanide ions with SDS micelles have been used for studying magnetic field effects on geminate pair recombination of radicals. ${ }^{48}$ In addition, there is increasing interest in the mesomorphism of lanthanide salts of anionic amphiphiles. ${ }^{49-51}$ 
We have, therefore, carried out a detailed study of the association of some trivalent lanthanide ions with sodium dodecyl sulfate micelles in aqueous solutions using their luminescence. This is complemented by results from other techniques. In contrast to previous studies, ${ }^{43,45}$ we will use the hydrated lanthanide ions directly, such that the results will also help understanding the ion binding of other hydrated divalent and trivalent cations.

\section{Experimental Section}

Reagents. Cerium(III), terbium(III), and europium perchlorates (Aldrich) and sodium dodecyl sulfate (Fluka puriss) were of the purest grade available and were used as received. Lanthanide(III) perchlorate salts were used, as these show only limited tendency for ion pair formation in solution. ${ }^{52}$ Deuterium oxide (99.9 at. \%) from Aldrich was used in preparing solutions for determining the number of coordinated water molecules. Gadolinium(III) perchlorate was prepared by dissolving the oxide in perchloric acid and diluting with water. Solutions were prepared using Millipore-Q water. Trivalent lanthanide ions do hydrolyze at high $\mathrm{pH}^{53}$ and, where necessary, $\mathrm{pH}$ (ca. 5) was adjusted by adding perchloric acid.

\section{Apparatus and Methods}

Potentiometric titrations were carried out using an Orion sodium ion selective electrode with a calomel reference electrode and a Metrohm $713 \mathrm{pH}$ meter. Temperature was kept at $25^{\circ} \mathrm{C}$, and the system was calibrated using different concentrations (1 $\mathrm{mM}-0.1 \mathrm{M}$ ) of aqueous solutions of sodium chloride. A good Nernstian response of the electrode was observed over this range. For studies on micellar solutions, ionic strength was not controlled, as addition of salts is known to affect the aggregation behavior of SDS micelles in solution. ${ }^{54}$ However, the high charge on the micelles and the lanthanide ions implies that the ionic strength will be high, and that effects resulting from this on the potentiometric titrations cannot be ignored. Absorption spectral measurements were made in $1 \mathrm{~cm}$ quartz cuvettes on a Shimadzu UV-2100 spectrophotometer. For luminescence spectral measurements, Spex Fluorolog 111 and Jobin-Ivon-Spex Fluorolog 3-22 instruments were used in right-angle configuration. In energy transfer experiments between $\mathrm{Ce}(\mathrm{III})$ and $\mathrm{Tb}$ (III) an appropriate filter was introduced in front of the emission monochromator to eliminate higher order bands. For variable temperature measurements, the temperature of the sample holder was controlled using water circulating from a thermostated bath. Tb(III) luminescence lifetimes were measured using the Spex 1934D phosphorimeter accessory with the Fluorolog 3-22 instrument, and decays were analyzed by using the program Origin 5.0 (Microcal). Static light scattering measurements were carried out using a spectrofluorimeter and a method described elsewhere. ${ }^{55}$ This involved using the Spex 111 fluorimeter in $90^{\circ}$ configuration with the excitation monochromator set at $436 \mathrm{~nm}$, and the emission spectrum scanned through this region. $0.5 \mathrm{~mm}$ excitation and $1.25 \mathrm{~mm}$ emission slits were used. Electron paramagnetic resonance (EPR) measurements were made in aqueous solutions using samples in the sealed capillary part of Pasteur pipets, on a Bruker EMX spectrometer. All spectra were corrected for the weak paramagnetic background in these pipets by using blank samples. The experimental setup for flash photolysis experiments comprises a Spectra-Physics Quanta-Ray GCR-130 Nd:YAG laser, an Applied Photophysics LKS 60 spectrometer, and a HewlettPackard Infinium oscilloscope (500 MHz, $1 \mathrm{Gsa} \mathrm{s}^{-1}$ ). The fourth harmonic $(266 \mathrm{~nm})$ of the laser was used for excitation, and

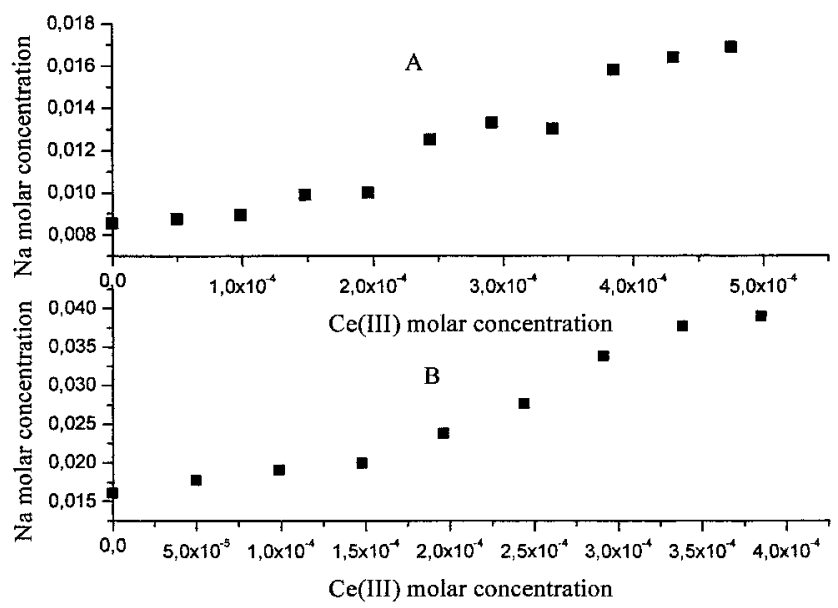

Figure 1. Change in free $\mathrm{Na}^{+}$concentration upon addition of different amounts of Ce(III) in samples with SDS $8 \mathrm{mM}$ (A) and $15 \mathrm{mM}$ (B) followed by Na potentiometric titration.

the cerium(III) emission was monitored at $360 \mathrm{~nm}$ using a Hamamtsu IP-28 photomultiplier.

\section{Results}

Initial visual observations were made on the phase behavior of aqueous solutions of SDS in the presence of lanthanide ions. Upon addition of cerium(III) perchlorate to an aqueous solution of SDS $(10 \mathrm{mM})$ at room temperature $\left(\approx 20{ }^{\circ} \mathrm{C}\right)$, formation of a precipitate was observed at lanthanide concentrations above about $5 \times 10^{-4} \mathrm{M}$. Upon increasing the SDS concentration to $20 \mathrm{mM}$, the concentration of lanthanide needed to induce precipitation was seen to increase. In a separate experiment, SDS was added to solutions of cerium(III) or terbium(III) perchlorate $\left(2 \times 10^{-4} \mathrm{M}\right)$. In both cases, turbidity was observed with SDS concentrations in the range 8 to $9 \times 10^{-4} \mathrm{M}$. Above the SDS critical micelle concentration $\left(\mathrm{cmc}, 8 \times 10^{-3} \mathrm{M}\right.$ ), clear, isotropic solutions were observed.

Further information on the interaction of lanthanide ions with the surfactant solutions was obtained by potentiometric titration using a sodium ion selective electrode. Difficulties were observed in obtaining quantitative data due to instabilities in the system, which resulted in a drift in potential with time in the presence of SDS. This may be due to adsorption of the surfactant by the sodium selective or reference electrode, and hence it is possible to obtain only qualitative information. However, by making measurements at constant time after addition of solution, marked increases in the free sodium ion concentration were observed when cerium(III) was added to aqueous solutions of SDS ( 8 and $15 \mathrm{mM}$, Figure 1, parts a and b), clearly showing ion exchange of $\mathrm{Na}^{+}$by $\mathrm{Ce}^{3+}$ in the micelles. However, the quantitative ion exchange constants $(\approx 10-100)$ are much greater than would be expected from simple electrostatic arguments. We believe that both surfactant adsorption by the membrane and ionic strength effects may be important. Future experiments will focus on this and attempt to explain the observed behavior.

The effect of adding lanthanide ions on the micellar structure was monitored by static light scattering. Upon addition of various concentrations of cerium(III) perchlorate to a $10 \mathrm{mM}$ solution of SDS, a small, gradual increase in scattering intensity was observed (Figure 2). This suggests that there may be a very slight increase in micellar size or change in shape over this concentration range on adding lanthanide ions. This is in agreement with the work of Almgren and Swarup, ${ }^{56}$ who showed by static fluorescence quenching for average surfactant con- 


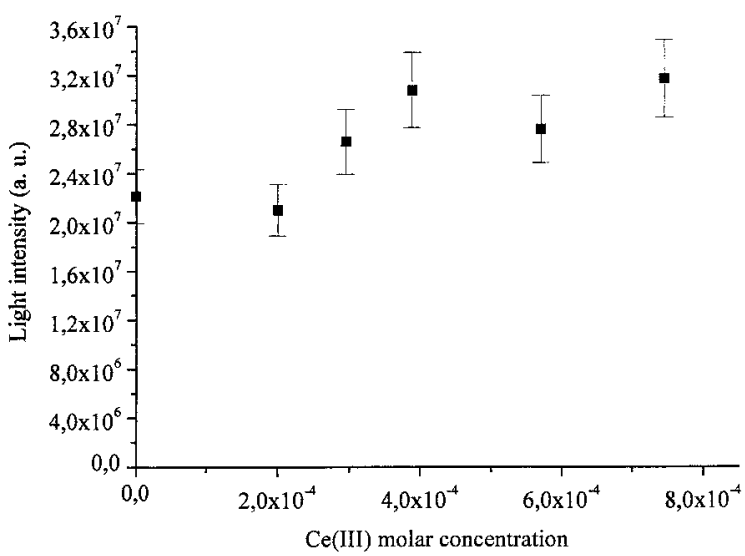

Figure 2. Gradual increase of light scattering upon addition of Ce(III) to a $10 \mathrm{mM}$ SDS solution.

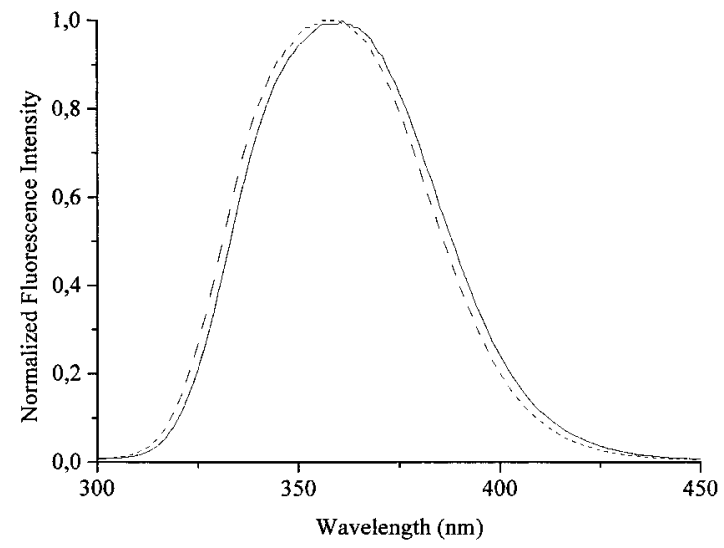

Figure 3. Normalized Ce(III) emission spectra in aqueous solution (solid line) and in the presence of SDS $(10 \mathrm{mM})$ (dashed line).

centration $44 \mathrm{mM}$ that the aggregation number of SDS micelles increased from 69 to about 81 when $\mathrm{Tb}^{3+}$ concentrations from 2 to $10 \mathrm{mM}$ were added.

Information on this system at a molecular level was obtained by studying the emission spectrum of aqueous solutions of cerium(III) perchlorate. In the presence of SDS (20 mM), a small, but significant, blue shift was observed in the emission maximum (Figure 3). While differences can be observed between emission spectra of solutions in pure water and in the presence of micellar aggregates due to light scattering, these will lead to red shifts. ${ }^{57}$ The observed blue shift suggests that there is a weak interaction between the lanthanide and the surfactant, with cerium(III) probably lying close to the sulfate headgroups.

Further information on lanthanide-surfactant interactions was obtained by studying its effect on the decay of lanthanide ion luminescence. The study of $\mathrm{Tb}$ (III) or $\mathrm{Eu}$ (III) luminescence decay in aqueous and $\mathrm{D}_{2} \mathrm{O}$ solutions provides a valuable method for measuring the number of coordinated water molecules. ${ }^{29,58}$ The decay of $\mathrm{Tb}(\mathrm{III})$ luminescence was studied alone (([Tb(III)] $\left.=10^{-2} \mathrm{M}\right)$ and in the presence of SDS $\left([\mathrm{Tb}(\mathrm{III})]=2 \times 10^{-4}\right.$ $\mathrm{M}$, [SDS] $=10 \mathrm{mM}$ ) in $\mathrm{H}_{2} \mathrm{O}$ and $\mathrm{D}_{2} \mathrm{O}$ solutions. Good exponential decays were observed in all cases, from which lifetimes and decay constants were calculated (Table 1). From this, the number of bound water molecules was determined. The values for aqueous solution are in good agreement with literature data $^{29}$ and are consistent with $\mathrm{Tb}$ (III) being coordinated to 9 water molecules. However, with the solution in the presence of SDS, the number of bound water molecules appears to decrease to approximately 8 . Some measurements were also made on
TABLE 1: Lifetime, $\tau$, Decay Constant, $K$, and Number of Coordinated Water Molecules (when possible), $n$, of $\mathbf{T b}(\mathrm{III})$, $\mathrm{Eu}(\mathrm{III})$, and $\mathrm{Ce}(\mathrm{III})$ in Water and $\mathrm{D}_{2} \mathrm{O}$ and in SDS Aqueous and $\mathrm{D}_{2} \mathrm{O}$ Solutions

\begin{tabular}{llcc}
\hline \multicolumn{1}{c}{ sample } & $\tau(\mathrm{ms})$ & $\mathrm{k}\left(\mathrm{ms}^{-1}\right)$ & $\mathrm{n}$ \\
\hline $\mathrm{Tb}\left(\mathrm{H}_{2} \mathrm{O}\right)$ & 0.43 & 2.5 & 9 \\
$\mathrm{~Tb}\left(\mathrm{D}_{2} \mathrm{O}\right)$ & 4.02 & 0.2 & \\
$\mathrm{~Tb}\left(\mathrm{SDS}+\mathrm{H}_{2} \mathrm{O}\right)$ & 0.45 & 2.5 & 8 \\
$\mathrm{~Tb}\left(\mathrm{SDS}+\mathrm{D}_{2} \mathrm{O}\right)$ & 2.29 & 0.4 & \\
$\mathrm{Eu}\left(\mathrm{H}_{2} \mathrm{O}\right)$ & $0.11^{*}$ & 10 & 9 \\
$\mathrm{Eu}\left(\mathrm{D}_{2} \mathrm{O}\right)$ & 3.30 & 0.3 & \\
$\mathrm{Eu}\left(\mathrm{SDS}+\mathrm{H}_{2} \mathrm{O}\right)$ & $0.11^{a}$ & 10 & - \\
$\mathrm{Eu}\left(\mathrm{SDS}+\mathrm{D}_{2} \mathrm{O}\right)$ & 1.78 & 0.5 & \\
$\mathrm{Ce}\left(\mathrm{H}_{2} \mathrm{O}\right)$ & $4.61 \mathrm{e}-5$ & $2.2 \mathrm{e} 4$ & - \\
$\mathrm{Ce}\left(\mathrm{D}_{2} \mathrm{O}\right)$ & $4.52 \mathrm{e}-5$ & $2.2 \mathrm{e} 4$ & \\
$\mathrm{Ce}\left(\mathrm{SDS}+\mathrm{H}_{2} \mathrm{O}\right)$ & $4.46 \mathrm{e}-5$ & $2.2 \mathrm{e} 4$ & - \\
$\mathrm{Ce}\left(\mathrm{SDS}+\mathrm{D}_{2} \mathrm{O}\right)$ & $4.35 \mathrm{e}-5$ & $2.3 \mathrm{e} 4$ &
\end{tabular}

${ }^{a}$ This is at the limit of time resolution of the phosphorimeter.

the decay of europium(III) luminescence in the presence $\left([\mathrm{Eu}(\mathrm{III})]=4 \times 10^{-4} \mathrm{M},[\mathrm{SDS}]=10 \mathrm{mM}\right)$ and absence of SDS $([\mathrm{Eu}(\mathrm{III})]=10 \mathrm{mM})$, and are included in Table 1. Although, since lifetimes in $\mathrm{H}_{2} \mathrm{O}$ are close to the time resolution of our system, it is not possible to determine the number of coordinated water molecules, the decrease in lifetime in $\mathrm{D}_{2} \mathrm{O}$ in the presence of SDS compared with the pure solvent is, again, consistent with a decrease in the number of water molecules bound to the lanthanide ion on interaction with the SDS micelles. Decreases in the number of coordinated water molecules have also been observed on binding of lanthanides to the polyelectrolyte poly(vinyl sulfate) in aqueous solutions ${ }^{59}$ and to the sulfonate headgroups of the surfactant AOT (bis(2-ethylhexyl)sulfosuccinate) in AOT/water/cyclohexane microemulsions. ${ }^{60}$ Measurements were also carried out on the lifetime of cerium(III) in the SDS system, and are presented in Table 1 . The lifetime in water is in excellent agreement with literature data. ${ }^{39}$ As expected for the $5 \mathrm{~d} \rightarrow 4 \mathrm{f}$ transition involved in this emission, no significant effect of deuteration of the solvent was observed on the lifetimes. However, there appeared to be a small decrease in lifetime in the presence of SDS, again in agreement with binding of the lanthanide ion by the micelle.

Electron paramagnetic resonance spectroscopy has been shown to be a good technique for studying ion binding to micelles. ${ }^{19}$ While $\mathrm{Ce}(\mathrm{III}), \mathrm{Tb}(\mathrm{III})$, and Eu(III) do not normally give EPR signals at room temperature due to their short relaxation times (typically $10^{-13} \mathrm{~s}$ ), and consequent line broadening, reasonable signals can be obtained with gadolinium(III), which has a much longer relaxation time $\left(10^{-9}-10^{-10} \mathrm{~s}\right){ }^{61-63}$ The EPR spectra were recorded of aqueous solutions of Gd(III) (1 $\mathrm{mM}$ ) in water and with various concentrations of SDS. A broad singlet was observed in all cases (Figure 4). However, the line width of this was found to only increase very slightly $(\leq 10 \%)$ in the presence of the surfactant, and to be virtually independent of SDS concentration. This supports the idea that the hydrated $\mathrm{Gd}(\mathrm{III})$ is close to the micellar surface, but still has reasonably free rotational motion. This description is consistent with results previously reported based on the observation of the effect of Gd(III) on water proton-relaxation times in SDS micelles. ${ }^{17}$ EPR $^{64}$ and time-resolved fluorescence quenching ${ }^{65}$ studies on copper(II) with SDS micelles suggest that in addition to their free rotational motion in these systems, polyvalent cations also have considerable lateral mobility on the micellar surface.

Further information on ion binding to amphiphile aggregates can be obtained by studying energy transfer. ${ }^{43,45} \mathrm{Ce}$ (III) and $\mathrm{Tb}$ (III) can normally only undergo electronic energy transfer at relatively short distances. ${ }^{40,45}$ Although the cerium(III) absorp- 


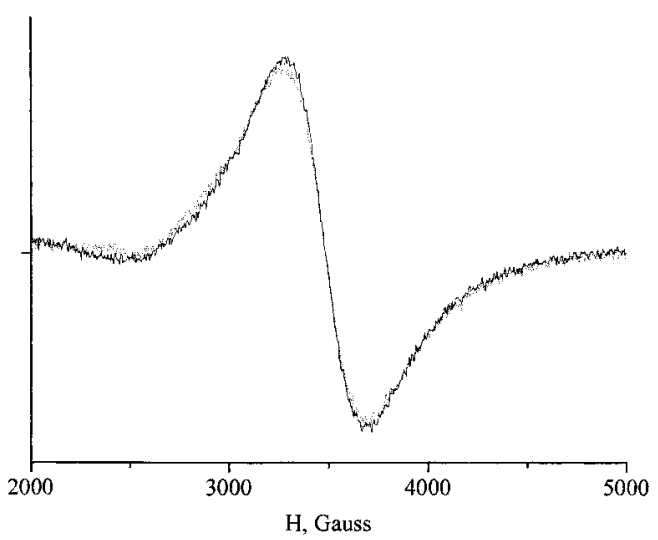

Figure 4. EPR spectra of $\mathrm{Gd}(\mathrm{III})(1 \mathrm{mM})$ in aqueous solution, solid line, and in SDS solution (2.5e-2 M) dashed line.

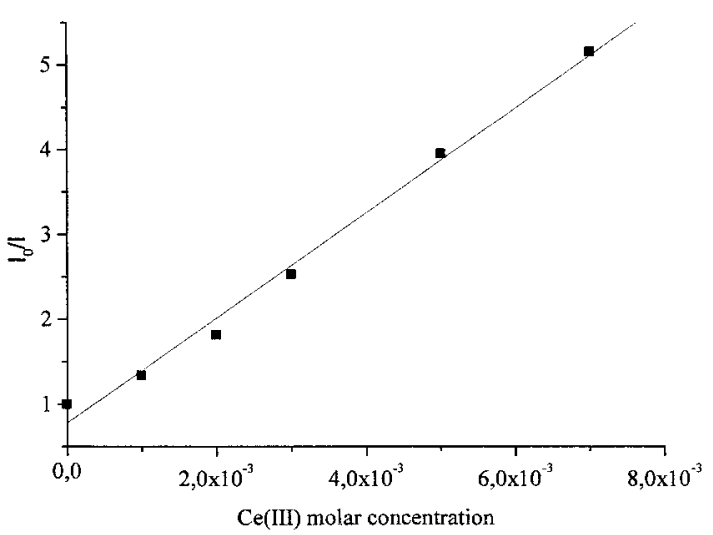

Figure 5. Stern-Volmer plot for relative $\mathrm{Tb}(\mathrm{III})$ emission intensity $\left(I_{0} / l\right)$ versus $\mathrm{Ce}(\mathrm{III})$ concentration in aqueous solution.

tion spectrum overlaps with part of that of $\mathrm{Tb}(\mathrm{III})$, the cerium(III) transition is fully allowed, and has a much higher molar absorption coefficient. Therefore, the majority of light will be absorbed by $\mathrm{Ce}(\mathrm{III})$ ions. Upon excitation of aqueous solutions of cerium(III) and terbium(III) $(5 \mathrm{mM})$ at $258 \mathrm{~nm}$, a weak emission of $\mathrm{Tb}(\mathrm{III})$ was observed in the $500-650 \mathrm{~nm}$ region. ${ }^{66}$ Most of this was expected to result from direct excitation of the terbium. Confirmation of the lack of energy transfer came from a flash photolysis study of the decay of luminescence of a solution of cerium(III) $(1 \mathrm{mM})$ in the presence of varying concentrations of $\mathrm{Tb}(\mathrm{III})$. The excited $\mathrm{Ce}$ (III) lifetime $(46.1 \pm 0.35)$ ns was independent of $\mathrm{Tb}$ (III) concentration up to the highest concentration studied $(20 \mathrm{mM})$, showing that $\mathrm{Ce}(\mathrm{III}) \rightarrow \mathrm{Tb}$ (III) electronic energy transfer in aqueous solutions is inefficient within the concentration region studied. A surprising result was that when the luminescence was studied by exciting the $\mathrm{Tb}(\mathrm{III})(10 \mathrm{mM})$ emission directly, this was seen to be quenched by cerium(III). A Stern-Volmer plot is given in Figure 5. While this process was not studied in detail, it is possible that it may either involve quenching by some low lying $f-f$ transition of $\mathrm{Ce}(\mathrm{III})$ or some charge transfer interaction between the metal ions, as has previously been reported for cerium(III) in the solid state. ${ }^{38}$

Energy transfer between cerium(III) and Tb(III) was studied in SDS micelles. The lack of energy transfer in aqueous solution and the short lifetime of cerium(III) excited state (ca. $50 \mathrm{~ns}$ ) compared with the time for collision between micelles ( $>1 \mu \mathrm{s}$ assuming that it is diffusion controlled) indicates that if energy transfer is observed, it can only arise from the two lanthanide ions already being present on the same micelle. Aqueous solutions of cerium(III) $\left(2 \times 10^{-4} \mathrm{M}\right)$ and SDS $(8-15 \mathrm{mM})$

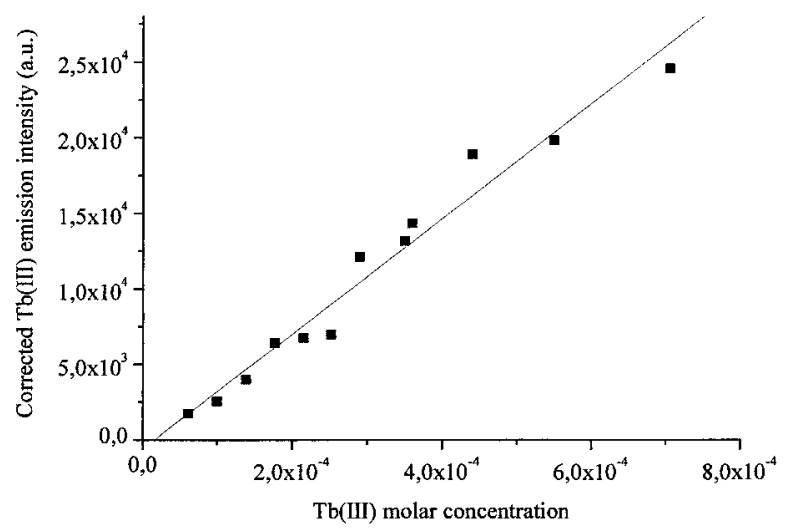

Figure 6. Corrected $\mathrm{Tb}$ (III) emission intensity versus $\mathrm{Tb}$ (III) molar concentration in SDS (1e-2 M) aqueous solution with Ce(III) 2e-4 M.

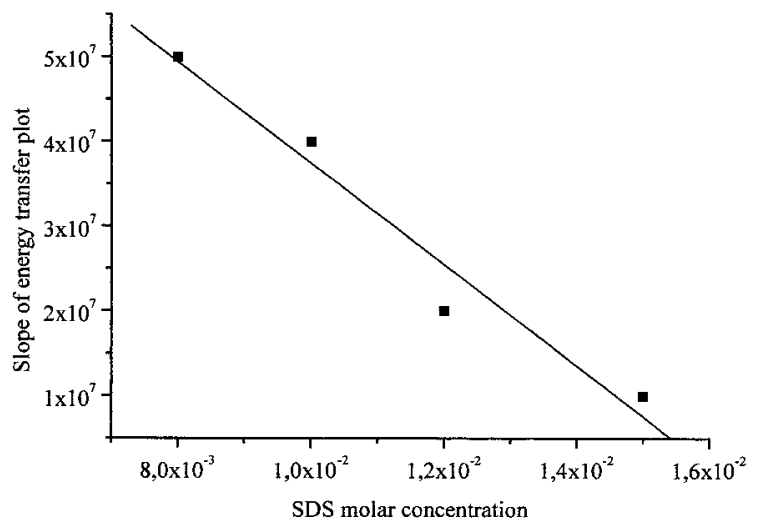

Figure 7. Slope of energy transfer plot versus SDS molar concentration.

were excited at $258 \mathrm{~nm}$ in the presence of $\mathrm{Tb}(\mathrm{III})\left(6 \times 10^{-5}-7\right.$ $\times 10^{-4} \mathrm{M}$ ), and the $\mathrm{Tb}(\mathrm{III})$ emission monitored at $542 \mathrm{~nm}$. Blank experiments were carried out under identical conditions in the absence of $\mathrm{Ce}$ (III), and the emission due to direct excitation of terbium subtracted. The corrected terbium emission for $10 \mathrm{mM}$ SDS solution is plotted as a function of terbium concentration in Figure 6. Although there seems to be slight curvature at the both low and high $\mathrm{Tb}$ concentrations, a general linear trend is observed at intermediate concentrations. The slopes of these plots are found to decrease with increasing SDS concentration as can be seen in Figure 7. This energy transfer may either occur via a collisional quenching of excited cerium(III) by terbium(III), as is commonly observed in triplettriplet energy transfer in organic molecules, ${ }^{67}$ or by a static process involving lanthanide ions located at an appropriate donor-acceptor separation, as has been reported for cerium(III) and terbium(III) adsorbed on porous glass. ${ }^{68}$ Studies on the dynamic quenching of excited terbium(III) bound to SDS micelles by $\mathrm{NO}_{2}{ }^{-}$ions show a rate constant $1.1 \times 10^{7} \mathrm{M}^{-1}$ $\mathrm{s}^{-1}$, which is 2 orders of magnitude less than that in free solution. ${ }^{69}$ If this is diffusion controlled, the rate between two trivalent ions with the same charge will be even lower. Considering that the lifetime of excited cerium(III) is about 45 $\mathrm{ns}$, the probability of collision between this and $\mathrm{Tb}^{3+}$ during its lifetime will be extremely small for the ion concentrations used. We therefore favor a static mechanism.

A rationale for the experimental findings has been sought through the configurational characterization of the systems studied obtained via Monte Carlo simulation with the Metropolis algorithm, ${ }^{70}$ utilizing the MOLSIM package. ${ }^{71}$ The model comprises a set of charged hard spheres representing the micelle, the terbium(III) and cerium(III) ions, and the SDS and salt 
TABLE 2: Characteristics of Hard-Sphere Particles in Model Systems

\begin{tabular}{lcllll}
\hline & micelle & $\mathrm{Ce}^{3+}$ & $\mathrm{Tb}^{3+}$ & $\mathrm{Na}^{+}$ & $\mathrm{ClO}_{4}^{-}$ \\
\hline radius $^{a} / \AA$ & 15 & 2.61 & 2.50 & 0.95 & 3.5 \\
charge/e & -64 & 3 & 3 & 1 & -1
\end{tabular}

${ }^{a}$ Including approximation to first solvation sphere.

TABLE 3: Characteristics of Systems Studied for Each Value of the SDS Concentration

\begin{tabular}{cccccc}
\hline $\begin{array}{l}\text { boundary } \\
\text { radius / }\end{array}$ & $n$ mic & $n \mathrm{Na}^{+}$ & $n \mathrm{Ce}^{3+}$ & $n \mathrm{~Tb}^{3+}$ & $n \mathrm{ClO}_{4}^{-}$ \\
\hline 159.1 & & 2 & 2 & 12 \\
& & & 4 & 18 \\
& & & 6 & 24 \\
& & & 8 & 30 \\
182.1 & \multirow{3}{*}{64} & 3 & 2 & 15 \\
& & & 5 & 24 \\
& & & 11 & 33 \\
& & & 2 & 42 \\
& & & 4 & 33 \\
230.0 & & & 7 & 42 \\
& & & 11 & 54 \\
& & & 14 & 63 \\
& & & 16 & 69
\end{tabular}

counterions (sodium and perchlorate, respectively). As has been discussed by Woodward and Jönsson, ${ }^{72}$ in systems such as this, where high valent counterions or high charge densities are present, approximations such as the direct use of the PoissonBoltzmann method cannot be employed. In the present calculations, only hard-sphere respulsions and Coulombic interactions on a continuum solvent with relative dielectric permittivity 78.4, equivalent to water, were considered. Tables 2 and 3 summarize the characteristics of the system. Micellar concentrations have been calculated from the total SDS concentration taking $[$ micelle $]=\left([\mathrm{SDS}]_{\text {total }}-\mathrm{cmc}\right) / N_{\text {agg }}$ taking a cmc value of $8 \times$ $10^{-3} \mathrm{M}$ and aggregation number $N_{\text {agg }}=64 .{ }^{73}$ For each SDS concentration, we have performed 4 or 5 calculations with varying terbium(III) concentration. Preliminary tests have indicated that the use of periodical boundary conditions does not significantly differ from what is found using a cell model with a spherical boundary, in which a single micelle is considered, with the number of other ions being established on the basis of relative concentrations. The volume of the cell is also changed appropriately. The results presented were thus obtained with this cell model.

In every case, irrespective of the lanthanide or counterion concentration, it is found from the calculations that the $\mathrm{Ce}$ (III) and $\mathrm{Tb}(\mathrm{III})$ ions are placed close to the micelle surface in the equilibrated systems, taking an average micelle radius of $15 \AA$ (see Table 2). A illustration of this fact can be found in the radial distribution functions of Figure 8 and the snapshot depicted in Figure 9. The calculations do not treat the hydration water explicitly, but from the radial distribution they suggest that when the lanthanide ions bind to the micellar surface there may be liberation of water molecules. This is consistent with the idea, based on luminescence lifetime measurements, that the lanthanide hydration number decreases on binding to the micelles.

The average distance $\langle r\rangle$ between the cerium and the closest terbium ions was estimated on the basis of running coordination numbers (rcn). This corresponds to the distance from each cerium which encompasses, on average, the closest terbium ion, i.e., a unit value for rcn. The $\mathrm{Tb}(\mathrm{III})$ emission arising from energy transfer from $\mathrm{Ce}(\mathrm{III})$, as monitored as its intensity at

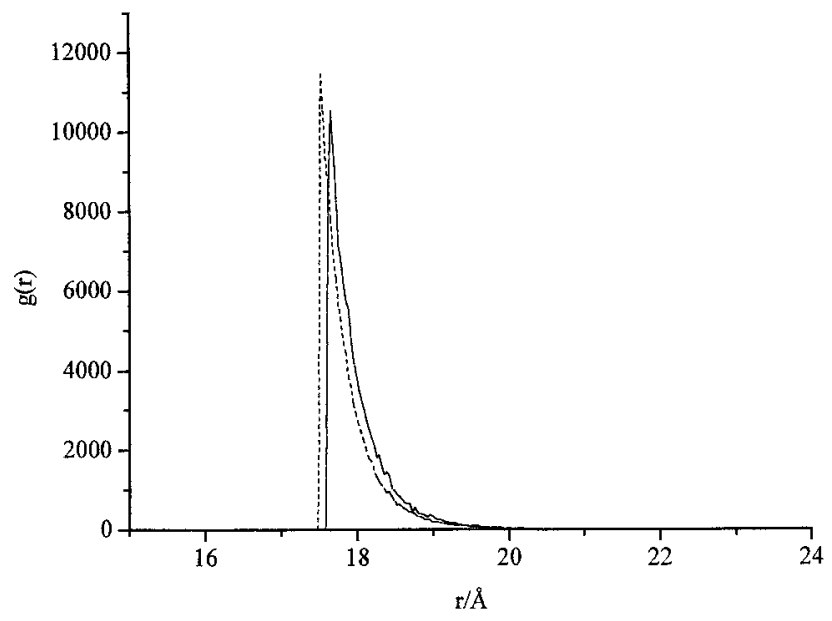

Figure 8. Micelle-lanthanide ion radial distribution functions, $g(r)$ : $\mathrm{Ce}^{3+}$, solid line; $\mathrm{Tb}^{3+}$, dashed line. Concentrations as follows: [Micelle] $=1 \times 10^{-4} \mathrm{M},\left[\mathrm{Ce}^{3+}\right]=2 \times 10^{-4} \mathrm{M},\left[\mathrm{Tb}^{3+}\right]=8 \times 10^{-4} \mathrm{M},\left[\mathrm{Na}^{+}\right]$ $=3 \times 10^{-3} \mathrm{M}$. The micellar concentration is calculated from the total surfactant concentration, the $\mathrm{cmc}$, and the average aggregation number as described in the text.

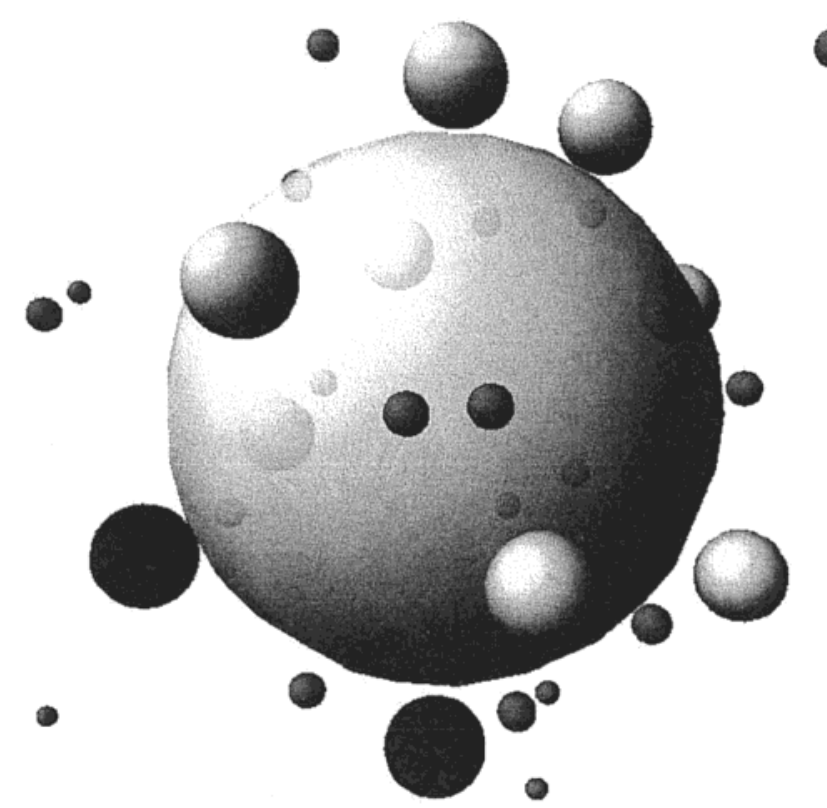

Figure 9. Snapshot of the equilibrated system showing the proximity of the micelle surface. The larger radii ions correspond to lanthanides and the smaller ones represent the sodium ions. Negative ions have been omitted for clarity. Concentrations as in Figure 8.

$542 \mathrm{~nm}$, depends on the average distance, $\langle r\rangle$. Both Dexter and Förster mechanisms have been suggested for energy transfer between lanthanide complexes in solution. ${ }^{40,43}$ These show different distance dependences. However, Förster transfer ${ }^{41}$ requires a good spectral overlap between the donor emission and acceptor absorption, and with hydrated terbium(III) in solution the low molar absorption coefficient makes this unfavorable. We therefore feel that a Dexter-type mechanism is more probable. A similar conclusion has been reached for energy transfer between $\mathrm{Ce}(\mathrm{III})$ and $\mathrm{Tb}$ (III) ions adsorbed on porous glass. ${ }^{68}$ This is expected to show a dependence on the inverse square of the donor-acceptor separation. In Figure 10 we have plotted some of the experimental intensity values in conjunction with curves that were obtained theoretically considering the terbium(III) emission intensity due to energy transfer(I) to be proportional to $\langle r\rangle^{-2}$. For all sets of data, the proportionality constant was set on the basis of a least squares 


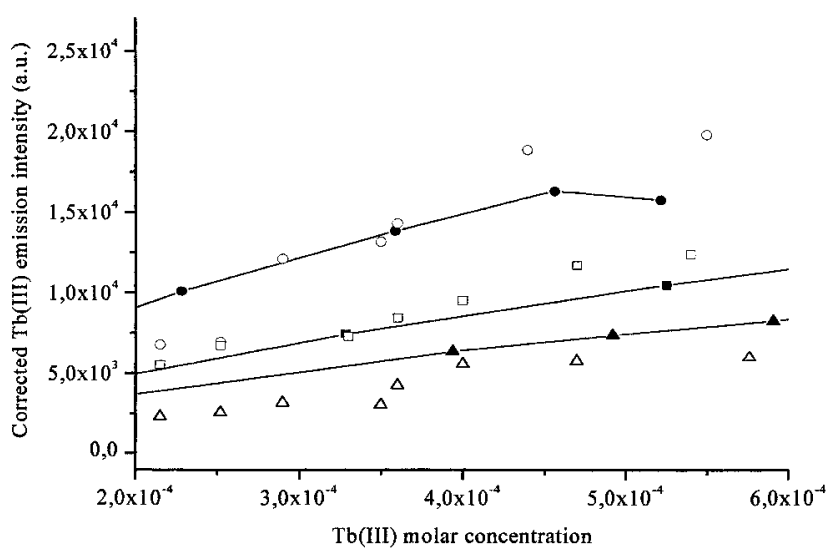

Figure 10. Scaled $1 /\langle r\rangle^{2}$ Monte Carlo results (full points, see text) plotted vs terbium(III) experimental emission intensity (open points). Calculated curves and experimental points from top to bottom correspond to SDS concentrations of $1.0 \times 10^{-2}, 1.2 \times 10^{-2}$, and $1.5 \times$ $10^{-2} \mathrm{M}$.

procedure following a linear interpolation of the experimental data. It is shown that the experimental trend is well reproduced, although quantitatively some discrepancy may be observed. According to the theoretical calculations, the intensity no longer increases when the total charge of the lanthanide ions present at the micelle surface is close to the charge of the micelle itself. This can be seen in Figure 10 for the lowest surfactant concentration.

All the calculations have been made considering a micelle of radius $15 \AA$. Additional calculations in which the radius was varied from 14 to $16 \AA$ show that, as might be anticipated, the calculated cerium(III) - terbium(III) average distances are sensitive to this parameter. The model is able to explain qualitatively the observed energy transfer and represents a good approach to analyze the problem. To obtain a more quantitative analysis, it is necessary to include aspects such as changes in micellar radius, aggregation number or shape stemming from the presence of the trivalent lanthanides, or reduction in the critical micelle concentration in the presence of polyvalent cations, which is suggested from studies on similar systems. ${ }^{11,74}$ Also, the assumption that energy transfer will simply depend on the inverse square of the donor-acceptor separation is an oversimplification, as higher order terms, resulting from dipoledipole, and other interactions are likely to be relevant. Future experimental studies will be directed toward the investigation of these effects.

\section{Discussion}

The interaction of lanthanide ions with sodium dodecyl sulfate micelles can be expected to mimic interactions of this surfactant with other polyvalent metal ions, such as calcium(II). Competing counterion binding to ionic micelles can conveniently be treated within an ion exchange framework. ${ }^{75}$ While it has not been possible to determine the ion exchange constant $K_{\mathrm{e}}$ (La(III)/ $\mathrm{Na}^{+}$), the potentiometric measurements using a sodium ion selective electrode suggest that this must be high. This is in agreement with the behavior reported for binding of other divalent $^{21,22,76,77}$ and trivalent ${ }^{21}$ ions to SDS. From the experimental and theoretical results presented in the present study it can be suggested that Coulombic interactions between the trivalent lanthanides and SDS surface play an important role in their binding, as seen from the blue shift of $\mathrm{Ce}(\mathrm{III})$ emission spectrum, the high degree of exchange of sodium by $\mathrm{Ce}(\mathrm{III})$ as shown by the potentiometric measurements, and the changes in $\mathrm{Tb}(\mathrm{III})$ and $\mathrm{Eu}(\mathrm{III})$ lifetimes in aqueous and $\mathrm{D}_{2} \mathrm{O}$ solutions in the presence and absence of SDS. Similarly, from the lifetime data, it is shown that this interaction results in the loss of at least one coordination water molecule of the lanthanide ions, and this is mimicked in Monte Carlo modeling studies by the close approach of the lanthanides to the SDS surface. However, from the Gd(III) EPR measurements there are no signs of the cations forming strong bonds to the sulfate groups, and the lanthanide ions still retain relatively high mobility.

When they are located at a relatively close distance, efficient electronic energy transfer is possible between cerium(III) and terbium(III) ions. In the present systems, this is only possible when they are present on the same micelle. This provides a very sensitive technique for showing the presence of multiple lanthanide ion binding to the micelles. The $\mathrm{Tb}(\mathrm{III})$ emission intensity upon energy transfer from cerium(III), corrected for direct excitation, increases approximately linearly with $\mathrm{Tb}$ (III) concentration, and the efficiency is also found to decrease with increasing SDS concentration. This is likely to be associated both with occupancy of the micelles by the lanthanide ions, and the $\mathrm{Ce}-\mathrm{Tb}$ distance on the surface of a single micelle. Although energy transfer between $\mathrm{Ce}(\mathrm{III})$ and $\mathrm{Tb}(\mathrm{III})$ is wellestablished there is still some controversy in the bibliography on the mechanism of this process. Both dipole-dipole (Förster) mechanism and an exchange (Dexter) mechanism have been proposed. ${ }^{40,45}$ For reasons discussed in the Results section, we feel that a Dexter-type mechanism is dominant. The energy transfer data has been further analyzed using a Monte Carlo simulation assuming a cell model, and the trends of the experimental data are qualitatively reproduced assuming that $\mathrm{Tb}$ emission intensity due to energy transfer is proportional to $\langle r\rangle^{-2}$, where $\langle r\rangle$ is the average distance between cerium and the closest terbium, as suggested from the Dexter model. ${ }^{42,68}$

\section{Conclusion}

In conclusion it has been shown that trivalent lanthanides show a higher tendency to associate with SDS micelles than monovalent cations. This association is due to electrostatic interactions and does not imply that any kind of bond between SDS and lanthanides is formed since ESR measurements on gadolinium(III) show that the cations, even in the proximity of the micelle surface, are freely rotating ions. However, both experimental results and modeling do show that the lanthanide ions lose at least one hydration water on binding to the micelles. This may have important implications in the modeling of counterion binding to charged micelles.

Energy transfer between $\mathrm{Ce}(\mathrm{III})$ and $\mathrm{Tb}(\mathrm{III})$ is possible when both ions are adsorbed on SDS surface and depends on SDS concentration, that is the concentration of micelles present. This would seem to provide a valuable tool for studying counterionmicelle association, and shows that each micelle is associated with several lanthanide ions.

Energy transfer can be successfully simulated via Monte Carlo simulation with a very simple model in which $\mathrm{Tb}$ emission intensity due to energy transfer is considered, as a first approximation, to be proportional to the inverse of square of the average distance between cerium and the closest terbium. Experimental results such as the higher affinity of trivalent ions for association with SDS relative to monovalent ions, the dependence of energy transfer on micelle size and the effect of $\mathrm{Tb}$ (III) concentration on energy transfer between $\mathrm{Ce}(\mathrm{III})$ and $\mathrm{Tb}$ (III) are well described qualitatively with this model. However, caution is needed in treating the energy transfer simply in terms of the inverse square of the cerium-terbium distance, 
and it is likely that for a more quantitative analysis it will be necessary to include higher order terms.

Acknowledgment. We are grateful to Carlos Serpa Soares for his assistance with flash photolysis studies, Prof. M. L. P. Leitão for her generous assistance with the potentiometric measurements, to Professors J. Laranjinha and C. F. G. C. Geraldes and J. Frade for their help in recording the EPR spectra, and to a referee for helpful comments. One of us (H.D.B.) also thanks Dr. A. Beeby for suggesting energy transfer as a possible mechanism of quenching of terbium(III) luminescence by cerium(III). Financial support from PRAXIS XXI, Sapiens/ POCTI and the award of a grant from the Universidad de Burgos (to M.J.T.) is gratefully acknowledged.

\section{References and Notes}

(1) Stigter, D. J. Phys. Chem. 1975, 79, 1008.

(2) Stigter, D. J. Phys. Chem. 1975, 79, 1015

(3) Gunnarsson, G.; Jönsson, B.; Wennerström, H. J. Phys. Chem. 1980 84, 3114.

(4) Evans, D. F.; Mitchell, D. J.; Ninham, B. W. J. Phys. Chem. 1984, $88,6344$.

(5) Evans, D. F.; Ninham, B. W. J. Phys. Chem. 1986, 90, 226.

(6) Brady, J. F.; Evans, D. F.; Warr, G. G.; Grieser, F.; Ninham, B.

W. J. Phys. Chem. 1986, 90, 1853.

(7) Evans, D. F.; Wennerström, H. The Colloidal Domain: Where Physics, Chemistry, Biology and Technology Meet; VCH: New York, 1994.

(8) Jönsson, B.; Lindman, B.; Holmberg, K.; Kronberg, B. Surfactants and Polymers in Aqueous Solution; Wiley: Chichester, 1998.

(9) Bunton, C. A.; Nome, F.; Quina, F. H.; Romsted, L. S. Acc. Chem. Res. 1991, 24, 357

(10) Miyamoto, S. Bull. Chem. Soc. Japan 1960, 33, 371.

(11) Miyamoto, S. Bull. Chem. Soc. Jpn. 1960, 33, 375.

(12) Hato, M.; Shinoda, K. J. Phys. Chem. 1973, 77, 378.

(13) Teppner, R.; Haage, K.; Wantke, D.; Motschmann, H. J. Phys. Chem. B 2000, 104, 11489.

(14) Bunton, C. A.; Ohmenzetter, K.; Sepulveda, L. J. Phys. Chem. 1977, $81,2000$.

(15) Cutler, S. G.; Meares, P.; Hall, D. G. J. Chem. Soc., Faraday Trans. $1 \mathbf{1 9 7 8}, 74,1758$.

(16) Rathman, J. F.; Scamehorn, J. F. J. Phys. Chem. 1984, 88, 5807.

(17) Robb, I. D. J. Colloid Interface Sci. 1971, 37, 521. 362 .

(18) Rosenholm, J. B.; Lindman, B. J. Colloid Interface Sci. 1976, 57,

(19) Stilbs, P.; Lindman, B. J. Colloid Interface Sci. 1974, 46, 177.

(20) Clifford, J.; Pethica, B. A. Trans. Faraday Soc. 1964, 60, 216.

(21) Hafiane, A.; Issid, I.; Lemordant, D. J. Colloid Interface Sci. 1991, $142,167$.

(22) Okano, L. T.; Alonso, E. O.; Waissbluth L.; O. L.; Quina, F. H. Photochem. Photobiol. 1996, 63, 746.

(23) Lanthanide Probes in Life, Chemical and Earth Sciences: Theory and Practice; Bünzli, J.-C. G., Choppin, G. R., Eds.; Elsevier: Amsterdam, 1989.

(24) Williams, R. J. P. Struct. Bonding 1982, 50, 79

(25) Horrocks, W. DeW., Jr.; Albin, M. Prog. Inorg.Chem. 1984, 31, 1.

(26) Frey, S. T.; Horrocks, W. DeW. Jr. Inorg. Chim. Acta 1995, 229 ,

(27) Chopin, G. R.; Wang, Z. M. Inorg. Chem. 1997, 36, 249.

(28) Stein, G.; Würzberg, E. J. Chem. Phys. 1975, 62, 208.

(29) Horrocks, W. DeW., Jr.; Sudnick, D. R. J. Am. Chem. Soc. 1979, $101,334$.

(30) Kalyanasundaram, K. Chem. Soc. Rev. 1978, 7, 453.

(31) Almgren, M. Adv. Colloid Interface Sci. 1992, 41, 9.

(32) Gehlen, M. H.; De Schryver, F. C. Chem. Rev. 1993, 93, 199.

(33) Zana, R. In Polymer-Surfactant Systems; Kwak, C. T., Ed.; Marcel Dekker: New York, 1998;Chapter 10.
(34) Miguel, M. da G.; Burrows, H. D.; Formosinho, S. J.; Lindman, B. J. Mol. Structure 2001, 563-564, 89 .

(35) Burrows, H. D.; Miguel, M. da G. Adv. Colloid Interface Sci. 2001, $89-90,485$.

(36) Carnall, W. T.; Fields, P. R.; Rajnak, K. J. Chem. Phys. 1968, 49 , 4447.

(37) Halladay, H. N.; Petersheim, M. Biochemistry 1988, 27, 2120.

(38) Blasse, G.; Schipper, W.; Hamelink, J. J. Inorg. Chim. Acta 1991, $189,77$.

(39) Okada, K.; Kaizu, Y.; Kobayashi, H.; Tanaka, K.; Marumo, F. Mol. Phys. 1985, 54, 1293.

(40) Frey, S. T.; Horrocks, W. DeW., Jr. Inorg. Chem. 1991, 30, 1073.

(41) Förster, T. Discuss. Faraday Soc. 1959, 27, 7.

(42) Dexter, D. L. J. Chem. Phys. 1953, 21, 836.

(43) Dong, W.; Flint, C. D. J. Chem. Soc., Faraday Trans. 1992, 88, 2661

(44) Neilson, J. D.; Shepherd, T. M. J. Chem. Soc., Faraday Trans. 2 1976, $72,557$.

(45) Dong, W.; Flint, C. D. J. Chem. Soc., Faraday Trans. 1992, 88, 3435 .

(46) Sun, J.; Petersheim, M. Biochim. Biophys. Acta 1990, 1024, 159

(47) Elbanowski, M.; Makowska, B. J. Photochem. Photobiol. A: Chemistry 1996, 99, 85.

(48) Turro, N. J.; Lei, X.; Gould, I. R.; Zimmt, M. B. Chem. Phys. Lett. 1985, $120,397$.

(49) Marques, E. F.; Burrows, H. D.; Miguel, M. da G. J. Chem. Soc., Faraday Trans. 1998, 94, 1729.

(50) Corkery, R. W.; Martin, J. P. D. J. Lumin. 1999, 82, 1

(51) Binnemans, K.; Jongen, L.; Görller-Walrand, C.; D’Olieslager, W.; Hinz, D.; Meyer, G. Eur. J. Inorg. Chem. 2000, 1429.

(52) Spedding, F. H.; Jaffe, S. J. Am. Chem. Soc. 1954, 76, 884.

(53) Moeller, T. J. Phys. Chem. 1946, 50, 242.

(54) Schott, H. J. Phys. Chem. 1966, 70, 2966.

(55) Mougán, M. A.; Coello, A.; Jover., A.; Meijide, F.; Tato, J. V. J. Chem. Educ. 1995, 72, 284.

(56) Almgren, M.; Swarup, S. J. Phys. Chem. 1983, 87, 876.

(57) Atherton, S. J.; Dymond, C. M. G. J. Phys. Chem. 1989, 93, 6809.

(58) Bünzli, J.-C. G. In Lanthanide Probes in Life, Chemical and Earth Sciences: Theory and Practice; Bünzli, J.-C. G., Choppin, G. R., Eds.; Elsevier: Amsterdam, 1989; Chapter 7.

(59) Tapia, M. J.; Burrows, H. D. Langmuir 2002, 18, 1872.

(60) Beeby, A.; Clarkson, I. M.; Eastoe, J.; Faulkner, S.; Warne, B. Langmuir 1997, 13, 5816.

(61) Hyde, J. S.; Sarna, T. J. Chem. Phys. 1978, 68, 4439.

(62) Stephens, E. M.; Grisham, C. M. Biochemistry 1979, 18, 4876

(63) Stephens, E. M. In Lanthanide Probes in Life, Chemical and Earth Sciences: Theory and Practice; Bünzli, J.-C. G., Choppin, G. R., Eds.; Elsevier: Amsterdam, 1989; Chapter 6.

(64) Bales, B.; Stenland, C. J. Phys. Chem. 1995, 99, 15163.

(65) Bales, B.; Almgren, M. J. Phys. Chem. 1995, 99, 15153

(66) $\mathrm{Tb}$ (III) does show another emission band below $500 \mathrm{~nm}$. However, this was not normally observed in our experiments due to the presence of a cutoff filter.

(67) Bensasson, R.; Land, E. J. Trans. Faraday Soc. 1971, 67, 1904.

(68) Hazenkamp, M. F. Ph.D. Thesis, University of Utrecht, 1966, Chapter 2.

(69) Grieser, F. J. Phys. Chem. 1981, 85, 928

(70) Allen, M. P.; Tildesley, D. J. Computer Simulation of Liquids; Clarendon, Oxford, 1987.

(71) MOLSIM, Version 3.1.7.; Linse, P., Lund University, Sweden, 2001.

(72) Woodward, C. E.; Jönsson, B. Chem. Phys. 1991, 155, 207.

(73) Doughty, D. A. J. Phys. Chem. 1981, 85, 3545

(74) Oko, M. V.; Venable, R. L. J. Colloid Interface Sci. 1971, 35, 53.

(75) Quina, F. H.; Chaimovich, H. J. Phys. Chem. 1979, 83, 1844.

(76) Baumüller, W. H.; Hoffmann, H.; Ulbricht, W.; Tondre, C.; Zana, R. J. Colloid Interface Sci. 1979, 64, 418.

(77) Abdel-Kader, M. H.; Braun, A. M.; Paillous, N. J. Chem. Soc., Faraday Trans. 1 1985, 81, 245. 